\title{
Serum bilirubin and the risk of rheumatoid arthritis
}

\author{
Du Juping ${ }^{1, \#}$ | Yuan Yuan ${ }^{1, \# ~ \mid ~ C h e n ~ S h i y o n g ~}{ }^{1}$ | Li Jun ${ }^{1}$ | Zhou Xiuxiu ${ }^{2}$ | \\ Ying Haijian $^{3}$ | Shi Jianfeng ${ }^{3}$ | Shen Bo ${ }^{1,3}$
}

${ }^{1}$ Department of Clinical Laboratory, Taizhou Hospital of Zhejiang Province, Taizhou, Zhejiang Province, China

${ }^{2}$ Department of Clinical Laboratory, Linhai City of Traditional Chinese Hospital, Linhai, Zhejiang Province, China

${ }^{3}$ Department of Clinical Laboratory, Taizhou Hospital of Zhejiang Province, Affiliated Hospital of Wenzhou Medical College,

Taizhou, Zhejiang Province, China

\section{Correspondence}

Shen Bo, Department of Clinical Laboratory, Taizhou Hospital of Zhejiang Province, Linhai, Zhejiang, China.

Email: 1282437698@qq.com
Background: Oxidative stress and immune imbalance play an important role in the pathogenesis of rheumatoid arthritis (RA). Bilirubin is a powerful antioxidant and also regarded as immunomodulator. Increased evidence shows that bilirubin should be a protective factor for autoimmune disease. However, the relationship between bilirubin and RA remain unclear.

Methods: We analyzed serum bilirubin levels and other laboratory and clinical data in 130 RA patients (35 patients without any complications), 81 osteoarthritis (OA) patients and 96 healthy controls.

Results: Binary logistic regression adjusted by age and gender revealed that the levels of serum total, indirect bilirubin were significantly lower in RA patients, when compared with healthy controls $(P=.015, O R=0.767,95 \% \mathrm{Cl}=0.619-0.951 ; P=.010$, $\mathrm{OR}=0.664,95 \% \mathrm{Cl}=0.487-0.906$, respectively) or OA patients $(P=.000, \mathrm{OR}=0.763$, 95\% Cl=0.661-0.882; $P=.000, O R=0.656,95 \% \mathrm{Cl}=0.532-0.808$, respectively). A reduced trend of levels of bilirubin has been detected along with increased disease activity, despite with no significance $(P>.05)$. Spearman rank test further demonstrated that IgG and ESR were negative associated with total, indirect bilirubin, and albumin, prealbumin, APOA, HDL-C were positively associated with bilirubin.

Conclusions: In conclusion, the levels of serum bilirubins were decreased in RA, and decreased levels could be associated with IgG, albumin and inflammatory marker ESR.

KEYWORDS

bilirubin, oxidative stress, rheumatoid arthritis

\section{1 | INTRODUCTION}

Rheumatoid arthritis (RA) is a systemic autoimmune disease with unknown etiology and characterized by the infiltrating inflammatory cells and synovial fibroblasts in the joints, leading to irreversible destruction of cartilage and bone. It has been established that oxidative stress has distinct contribution to the RA inflammation and tissue damage. ${ }^{1-3}$ During inflammation, immune activated cells in the inflamed RA synovium could generate mass of reactive oxygen species (ROS) via the NADPH oxidase system. ${ }^{4}$ Excess ROS and ROS-oxidized production initial the proinflammatory responses in RA along with the damage to the lipids, proteins, and DNA, ${ }^{5}$ which leading to the production of autoantibodies. Overexpression ROS has been detected in the blood and

\#Du Juping and Yuan Yuan equally contributed to this study as first coauthors. synovial fluid of RA patients. Of these, 8-hydroxy-2'-deoxyguanosine (8-OHdG), which is a biomarker for oxidized DNA and ROS, has been found correlated with RA disease activity. ${ }^{6}$ Accumulating evidence show that antioxidants might be an effective therapy to alleviate the illness for RA. ${ }^{7,8}$ Thus, oxidative stress and inflammation might contribute to the major pathogenesis of RA in the disease initial and progression.

Bilirubin is a powerful antioxidant to the ability of scavenging ROS and also has anti-inflammatory and immune suppressive activities by inhibiting inflammatory cell proliferation, inducing reactive inflammatory cell apoptosis, reducing pro-inflammatory cytokines, and downregulating the expression of $\mathrm{MHC}-\mathrm{II} .{ }^{9,10}$ The antioxidant capacity of bilirubin is stronger than other antioxidants, such as ascorbic acid, catalase, and vitamin E. Lower serum concentration of bilirubin has been demonstrated in various chronic inflammatory diseases, including systemic lupus erythematosus, polymyositis, hypertension, and coronary 
artery calcification. ${ }^{11-13}$ However, to our knowledge, the role of serum bilirubin with RA has not been sufficiently investigated.

Hence, the aim of this retrospective cross-sectional study was to examine the role of decreased serum bilirubin as a risk factor for RA and the correlation with clinical and laboratory characteristics of RA.

\section{2 | MATERIALS AND METHODS}

\section{1 | Subjects}

One hundred fifty-two consecutive RA patients who fulfilled the 2010 criteria of American College of Rheumatology/European League Against Rheumatism and admitted to the Taizhou Hospital from January 2012 to December 2015 were enrolled in this retrospective study. Twenty-two patients with hepatobiliary, gallbladder, or hemolytic diseases that could have a change on the bilirubin level were excluded. Laboratory parameters and basic clinical characteristics including blood pressure, BMI, medication, smoking/drinking habits, disease duration, morning stiffness and history of hypertension, cardiovascular disease, diabetes, and pulmonary diseases were recorded from medical records. Based on the 28-joint disease activity score (DAS28), RA patients were divided into three groups: low activity group (DAS28<3.2), moderate activity group ( $3.2 \leq \mathrm{DAS} 28<5.1$ ), and high activity group (DAS28 $\geq 5.1$ ). In addition, a group of 81 hospitalized osteoarthritis (OA) patients met the ACR classification criteria of OA were also recruited as disease control group, since OA patients always present the same clinical symptoms with RA. Ninety-six healthy volunteers were randomly selected in this study. All the OA patients and healthy control were confirmed to have no family and personal history of autoimmune disease, cancers, liver disease, renal diseases, cardiovascular disease, or other inflammatory diseases. Since complications might be the important confounding factors that influence the levels of bilirubins, only 35 RA patients without any other diseases were included for comparing with OA patients and healthy controls $(\mathrm{HC})$. The study was approved by the institutional review board of Ethics Committee of the Taizhou Hospital of Zhejiang province, and all the participants signed the written informed consent.

\section{2 | Data analysis}

Since all continuous variables were non-normal distribution checked by Kolmogorov-Smirnov test, data were expressed as median (25th75th percentiles). Binary logistic regression analysis with adjusted by age and gender was carried out to compare the differences in the laboratory data and clinical characteristics between RA patients and OA/ $\mathrm{HC}$ group. Kruskal-Wallis $\mathrm{H}$ nonparametric test was used to compare three groups. The serum bilirubin was divided into two groups with the median as the cutoff. Then, univariate and multivariate logistic regressions were performed to determine the independent predictors to the change of serum bilirubin. The correlation between bilirubin and other laboratory or clinical data was assessed using Spearman's rank test. Statistical analysis was conducted by SPSS 22.0 software (SPSS Inc., Chicago, IL, USA), and scatter plot was performed using GraphPad Prism (GraphPad Prism Software Inc., San Diego, CA, USA). A $P$ value $<.05$ was considered statistically significant.

\section{3 | RESULTS}

\subsection{The serum bilirubin level in RA patients and OA patients or healthy controls}

Thirty-five RA patients without any complications (female $77.1 \%$, median age 59.0) were included in comparison with 96 healthy controls (female $45.8 \%$, median age 40.0 ) or 81 OA patients (female $42.0 \%$, median age 56.0). The median disease duration in RA was 5 years. We compared serum total bilirubin, direct bilirubin, indirect bilirubin, LDL-C, HDL-C, ESR, CRP, albumin, A/G, NEU, LYM, and BMI levels of RA patients with $\mathrm{OA}$ and healthy controls by binary logistic regression with adjustment of age and gender (Table 1). In the RA group, median total bilirubin was 7.5 (6.2-9.0) $\mu \mathrm{mol} / \mathrm{L}$. This was significantly lower than OA patients (median 10.6 [8.1-13.5]) and HC group (median 11.2 [9.5-13.5]), $P<.05$. In addition, indirect bilirubin was also significantly lower in RA patients than OA $(P<.001, \mathrm{OR}=0.656,95 \% \mathrm{Cl}=0.532-0.808)$ and $\mathrm{HC}(P<.010$, $\mathrm{OR}=0.664,95 \% \mathrm{Cl}=0.487-0.906$ ), whereas serum direct bilirubin had marginal significance between $\mathrm{RA}$ and $\mathrm{HC}$ groups $(P=.093, \mathrm{OR}=0.644$, 95\% $\mathrm{Cl}=0.386-1.076)$ but significant difference between $\mathrm{RA}$ and $\mathrm{OA}$ patients $(P=.035, \mathrm{OR}=0.678,95 \% \mathrm{Cl}=0.473-0.973$, respectively; Table 1 and Figure 1). Moreover, there were lower median levels of albumin and $A / G$, but higher ESR and NEU in RA patients than in OA patients and $H C$ group $(P<.001)$. Remarkably, 75 th percentiles of albumin and $A / G$ in RA group were much lower than these in $\mathrm{OA}$ and $\mathrm{HC}$ groups.

\subsection{Association of serum bilirubin levels with RA disease activity}

Based on DAS28, RA patients were classified into three groups. One patient due to lack of clinical data was excluded, and 129 RA patients remained for further analysis. When the three groups were compared, there were no significant differences detected for age, gender, smoke status, alcohol consumption, and duration (Table 2). Then, increased disease activity was accompanied by a decreasing trend in serum total bilirubin (median, 9.80 vs 7.45 vs $7.45 \mu \mathrm{mol} / \mathrm{L}$ ), indirect bilirubin (median, 6.70 vs 4.65 vs $4.90 \mu \mathrm{mol} / \mathrm{L}$ ), and direct bilirubin (median, 4.00 vs 2.50 vs $2.40 \mu \mathrm{mol} / \mathrm{L}$ ), but without significance detected. Moreover, larger proportion of morning stiffness was detected in high activity group. Then, we combined moderate activity RA patients with high activity group together (DAS28 $\geq 3.2$ ) and observed that the median indirect bilirubin in DAS28<3.2 group (median $6.70 \mu \mathrm{mol} / \mathrm{L}$ ) was significantly higher than that in DAS28 $\geq 3.2$ RA patients (median $4.90 \mu \mathrm{mol} / \mathrm{L} ; P=.03$ ), data not shown. However, we still failed to validate a similarly significant association in total bilirubin ( $P=.053)$ and direct bilirubin (0.269).

\subsection{Association of serum bilirubin levels with laboratory and clinical data in RA patients}

The median was determined to the cutoff value of serum bilirubin. We analyzed the association of total bilirubin $(\leq 7.5 />7.5)$, direct bilirubin $(\leq 2.5 />2.5)$, and indirect bilirubin $(\leq 5.0 />5.0)$ with laboratory and clinical variables by univariate logistic regression (Table 3 ). It was 
TABLE 1 Clinical and laboratory characteristics in rheumatoid arthritis (RA) patients, osteoarthritis (OA) patients, and healthy controls

\begin{tabular}{|c|c|c|c|c|c|c|c|}
\hline & $\begin{array}{l}\text { RA patients ( } 35 \\
\text { cases) }\end{array}$ & $\begin{array}{l}\text { Healthy control } \\
\text { (96 cases) }\end{array}$ & & & $\begin{array}{l}\text { OA patients (81 } \\
\text { cases) }\end{array}$ & & \\
\hline & Median (IQR) & Median (IQR) & $P_{\text {ad }}$ & $\mathrm{OR}_{\mathrm{ad}}(95 \% \mathrm{Cl})$ & Median (IQR) & $P_{\text {ad }}$ & $\mathrm{OR}_{\mathrm{ad}}(95 \% \mathrm{Cl})$ \\
\hline Age (y) & $59.0(51.0-67.0)$ & $40.0(30.3-49.0)$ & .000 & 1.201 (1.121-1.287) & $56.0(45.0-64.0)$ & .000 & $1.082(1.045-1.120)$ \\
\hline Female & 27 (77.1) & $44(45.8)$ & .001 & $0.100(0.026-0.381)$ & $34(42.0)$ & .000 & 1.068 (1.038-1.100) \\
\hline Total bilirubin $(\mu \mathrm{mol} / \mathrm{L})$ & $7.5(6.2-9.0)$ & $11.2(9.5-13.5)$ & .015 & $0.767(0.619-0.951)$ & $10.6(8.1-13.5)$ & .000 & $0.763(0.661-0.882)$ \\
\hline Indirect bilirubin $(\mu \mathrm{mol} / \mathrm{L})$ & $4.9(3.9-6.6)$ & $7.3(5.7-8.7)$ & .010 & $0.664(0.487-0.906)$ & $7.2(5.6-9.7)$ & .000 & $0.656(0.532-0.808)$ \\
\hline LDL-C (mmol/L) & $2.0(1.6-3.1)$ & $2.2(2.0-2.7)$ & .702 & $0.875(0.441-1.736)$ & $1.3(1.2-1.6)$ & .016 & $0.532(0.318-0.890)$ \\
\hline $\mathrm{HDL}-\mathrm{C}$ (mmol/L) & $1.2(0.9-1.4)$ & $1.3(1.2-1.6)$ & .115 & $0.211(0.030-1.463)$ & $2.7(1.9-3.2)$ & .006 & $0.125(0.028-0.552)$ \\
\hline $\operatorname{ESR}(\mathrm{mm} / \mathrm{h})$ & $76.0(41.0-104.0)$ & $9.0(5.0-12.0)$ & .014 & $2.252(1.182-4.293)$ & $44.0(39.5-46.1)$ & .000 & $1.089(1.057-1.121)$ \\
\hline BMI & $21.4(18.2-24.2)$ & $23.0(21.0-24.6)$ & .013 & $0.742(0.586-0.938)$ & & & \\
\hline
\end{tabular}

The analysis was adjusted by gender and age with binary logistic regression.
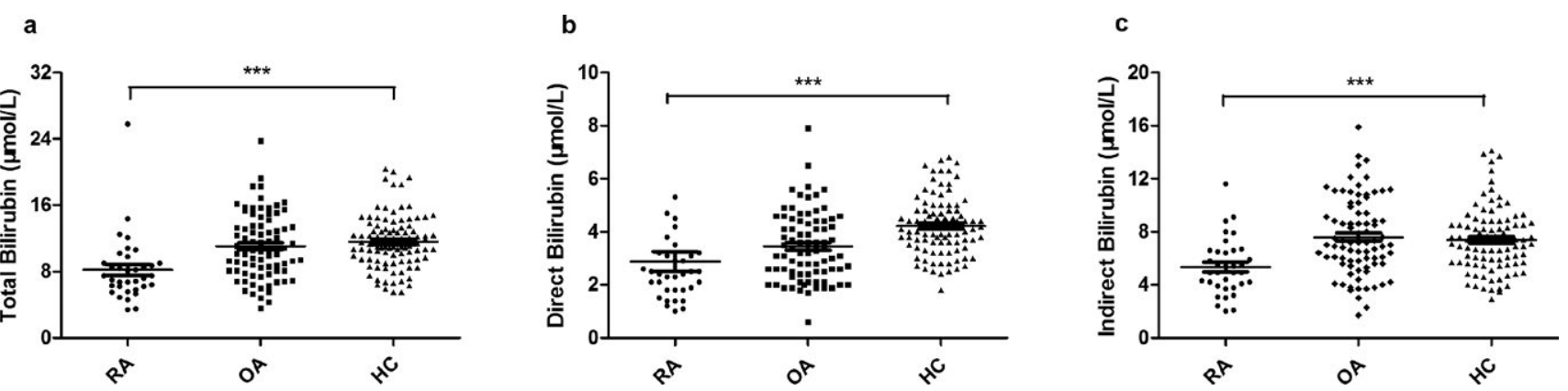

FIGURE 1 Analysis of serum bilirubin levels of rheumatoid arthritis patients ( $n=35)$, osteoarthritis patients ( $n=81$ ), and healthy controls $(\mathrm{n}=96)$. (a) Total bilirubin, (b) direct bilirubin, and (c) indirect bilirubin. ${ }^{* * *} P<.001$

found that higher total bilirubin was associated with higher $A / G$ and prealbumin. Those with higher indirect bilirubin had higher $A / G$, albumin, $\mathrm{CHE}$, and TBA, but lower prealbumin. Interestingly, those with higher IgG level had lower total bilirubin, direct bilirubin, and indirect bilirubin $(P<.05)$. Unfortunately, we failed to detect the similar association in RF and inflammatory markers (such as CRP and ESR).

Then, univariate variables with $P<.1$ were included in the multivariate logistic regression (Table 4). However, only prealbumin remained as an independent predictor for serum total bilirubin $(P=.033, \mathrm{OR}=1.166$, 95\% Cl=1.013-1.343) and indirect bilirubin $(P=.018, O R=1.212,95 \%$ $\mathrm{Cl}=1.034-1.421)$. Even more puzzling was that those with higher CRP levels had higher direct bilirubin, despite the OR value was really small $(P=.048, \mathrm{OR}=1.010,95 \% \mathrm{Cl}=1.000-1.019)$.

As shown in Table 5, the IgG levels of RA patients inversely correlated with serum total bilirubin, direct bilirubin, and indirect bilirubin ( $r=-.338, P=.001 ; r=-.230, P=.027 ; r=-.337, P=.001$, respectively). In addition, both total bilirubin and indirect bilirubin levels positively correlated with albumin, A/G, prealbumin, APOA, APOB, and HDL-C, but inversely correlated with ESR $(P<.05)$.

\section{DISCUSSION}

In this study, we demonstrated that serum bilirubin levels (including total, indirect, and direct bilirubins) were significantly lower in RA patients than osteoarthritis and healthy controls, especially in RA patients with high disease activity individuals. Furthermore, the relationship between serum bilirubin levels and some laboratory and clinical variables has been examined in RA patients.

Early in 1929, Philip Hench et al. made an interesting observation that the symptoms of rheumatoid arthritis relieved with the onset of jaundice or hyperbilirubinemia, indicating that high bilirubin might have the protect ability against the inflammatory diseases. ${ }^{14,15}$ Also, in a large epidemiologic study, serum total bilirubin has been confirmed to be associated with a decreased risk of rheumatoid arthritis. ${ }^{16}$ These results support our findings that serum bilirubin (including total, indirect, and direct bilirubin) has a significantly decreased level in RA patients relative to healthy controls, even to the osteoarthritis patients. Moreover, the decreased trend of serum bilirubin levels accompanied by increased the disease activity have been detected, albeit only 


\begin{tabular}{|c|c|c|c|}
\hline & DAS28<3.2 & DAS28 $=3.2-5.1$ & DAS28>5.1 \\
\hline Group & Median (IQR) & Median (IQR) & Median (IQR) \\
\hline Age $(y)$ & $59.0(46.0-69.5)$ & $58.5(54.0-67.0)$ & $65.0(59.0-70.5)$ \\
\hline Male & $2(22.2)$ & 19 (32.8) & $19(30.6)$ \\
\hline Smoke & $1(11.1)$ & $9(15.5)$ & $16(25.8)$ \\
\hline Alcohol consumption & $1(11.1)$ & $5(8.6)$ & $7(11.3)$ \\
\hline Morning stiffness & 7 (77.8) & $30(51.7)$ & $52(83.9)$ \\
\hline Duration (y) & $10.0(3.0-23.5)$ & $7.0(2.0-20.0)$ & $6.0(1.0-15.3)$ \\
\hline Charlson & $3.0(1.0-3.5)$ & $2.0(1.0-3.0)$ & $3.0(2.0-4.0)$ \\
\hline Total bilirubin $(\mu \mathrm{mol} / \mathrm{L})$ & $9.80(7.05-14.20)$ & $7.45(5.30-10.65)$ & $7.45(6.08-9.00)$ \\
\hline Direct bilirubin $(\mu \mathrm{mol} / \mathrm{L})$ & $4.00(1.80-5.35)$ & $2.50(1.50-3.53)$ & $2.40(1.88-3.20)$ \\
\hline Indirect bilirubin $(\mu \mathrm{mol} / \mathrm{L})$ & $6.70(5.00-8.85)$ & $4.65(3.30-7.40)$ & $4.90(3.70-5.90)$ \\
\hline
\end{tabular}

TABLE 2 The association between serum bilirubin levels and disease activity

TAB LE 3 Association between serum bilirubin levels and clinical and laboratory characteristics in rheumatoid arthritis patients

\begin{tabular}{|c|c|c|c|c|c|c|}
\hline & \multicolumn{2}{|c|}{ Total bilirubin $(\leq 7.5 />7.5 \mu \mathrm{mol} / \mathrm{L})$} & \multicolumn{2}{|c|}{ Direct bilirubin $(\leq 2.5 />2.5 \mu \mathrm{mol} / \mathrm{L})$} & \multicolumn{2}{|l|}{$\begin{array}{l}\text { Indirect bilirubin } \\
(\leq 5.0 />5.0 \mu \mathrm{mol} / \mathrm{L})\end{array}$} \\
\hline & OR & $P$ & OR & $P$ & OR & $P$ \\
\hline Age (y) & $0.988(0.954-1.023)$ & .488 & $0.972(0.938-1.006)$ & .108 & $0.997(0.963-1.032)$ & .868 \\
\hline Male & $1.397(0.661-2.951)$ & .381 & $1.045(0.496-2.202)$ & .907 & $1.156(0.548-2.435)$ & .704 \\
\hline Charlson score & $0.996(0.796-1.247)$ & .973 & $0.896(0.713-1.125)$ & .345 & $1.027(0.820-1.285)$ & .819 \\
\hline TBA $(\mu \mathrm{mol} / \mathrm{L})$ & $1.071(0.981-1.170)$ & .126 & $1.045(0.970-1.125)$ & .244 & $1.184(1.051-1.334)$ & .005 \\
\hline$A / G$ & $5.595(1.443-21.692)$ & .013 & $2.392(0.653-8.755)$ & .188 & $12.391(2.937-52.270)$ & .001 \\
\hline $\mathrm{CHE}(\mathrm{KU} / \mathrm{L})$ & $1.205(0.996-1.458)$ & .055 & $0.871(0.724-1.050)$ & .147 & $1.244(1.025-1.510)$ & .027 \\
\hline Ada (U/L) & $0.994(0.896-1.103)$ & .915 & $1.022(0.921-1.134)$ & .686 & $1.003(0.904-1.112)$ & .959 \\
\hline $\lg A(g / L)$ & $0.798(0.987-1.095)$ & .798 & $0.913(0.726-1.148)$ & .437 & $0.892(0.706-1.128)$ & .339 \\
\hline $\lg M(g / L)$ & $0.868(0.603-1.249)$ & .445 & $0.939(0.667-1.322)$ & .719 & $0.917(0.645-1.304)$ & .631 \\
\hline C3 (g/L) & $0.957(0.828-1.106)$ & .549 & $0.656(0.141-3.058)$ & .592 & $0.953(0.800-1.135)$ & .591 \\
\hline C4 (g/L) & $0.824(0.459-1.477)$ & .515 & $0.809(0.410-1.600)$ & .543 & $0.779(0.299-2.030)$ & .610 \\
\hline $\mathrm{RF}(\mathrm{KU} / \mathrm{L})$ & $1.000(0.999-1.000)$ & .582 & 1 & .828 & $1.000(0.999-1.000)$ & .711 \\
\hline CRP (mg/L) & $1.003(0.997-1.010)$ & .357 & $1.007(0.999-1.015)$ & .069 & 0.997 (0.991-1.004) & .451 \\
\hline Hypertension & $0.642(0.307-1.342)$ & .239 & $0.977(0.470-2.029)$ & .950 & $0.613(0.293-1.282)$ & .193 \\
\hline Lung disease & $1.330(0.602-2.939)$ & .480 & $1.130(0.513-2.491)$ & .761 & $1.277(0.578-2.820)$ & .546 \\
\hline Diabetes & $0.556(0.224-1.382)$ & .207 & $1.038(0.428-2.519)$ & .933 & $0.430(0.169-1.090)$ & .075 \\
\hline
\end{tabular}

indirect bilirubin has significantly difference between DAS $28 \leq 3.2$ and DAS28>3.2. It is consistent with previous findings that ROS, partially regulated by bilirubin, was positively associated with disease activity. The exact protective effect of bilirubin might be contributed to its potent antioxidative property for inhibiting oxidation damage from ROS that hampered the production of autoantibodies, and powerful immunomodulatory activity for inducing apoptosis of immune cells, down-regulating the expression of MHC-II molecules, and promoting Treg expansion. ${ }^{17,18}$ The HLA-DR4 has been discovered as the susceptibility factor for RA in large number of studies. ${ }^{10}$ Theoretical docking studies by Hideto Isogai revealed that bilirubin has the affinity to HLA-DR4 molecular, resulting in blocking the binding of 
TAB LE 4 Multiple logistic analysis the association between serum bilirubin levels and clinical and laboratory characteristics in rheumatoid arthritis patients

\begin{tabular}{|c|c|c|c|c|c|c|}
\hline & \multicolumn{2}{|l|}{ Total bilirubin } & \multicolumn{2}{|l|}{ Direct bilirubin } & \multicolumn{2}{|l|}{ Indirect bilirubin } \\
\hline Albumin (g/L) & - & - & - & - & $0.991(0.753-1.303)$ & .947 \\
\hline TBA ( $\mu \mathrm{mol} / \mathrm{L})$ & - & - & - & - & $1.443(0.926-2.249)$ & .105 \\
\hline$A / G$ & $0.140(0.002-8.057)$ & .342 & - & - & $0.150(0.000-175.287)$ & .598 \\
\hline $\lg G(g / L)$ & $0.875(0.743-1.031)$ & .111 & $0.944(0.878-1.014)$ & .114 & $0.908(0.711-1.160)$ & .442 \\
\hline $\mathrm{CRP}$ (mg/L) & - & - & $1.010(1.000-1.019)$ & .049 & - & - \\
\hline $\operatorname{ESR}(\mathrm{mm} / \mathrm{H})$ & - & - & $0.991(0.978-1.005)$ & .206 & - & - \\
\hline
\end{tabular}

TABLE 5 Correlation between serum bilirubins and laboratory data

\begin{tabular}{|c|c|c|c|c|c|c|}
\hline & \multicolumn{2}{|c|}{ Total bilirubin } & \multicolumn{2}{|c|}{ Direct bilirubin } & \multicolumn{2}{|c|}{ Indirect bilirubin } \\
\hline & $r$ & $P$ & $r$ & $P$ & $r$ & $P$ \\
\hline Charlson & .005 & .951 & -.061 & .488 & .044 & .621 \\
\hline Age (y) & -.106 & .232 & -.148 & .093 & -.058 & .514 \\
\hline Total protein $(\mathrm{g} / \mathrm{L})$ & -.091 & .307 & -.129 & .145 & -.051 & .566 \\
\hline Albumin (g/L) & .192 & .029 & .038 & .671 & .254 & .004 \\
\hline$A / G$ & .293 & .001 & .147 & .097 & .329 & .000 \\
\hline Prealbumin (mg/dL) & .357 & .004 & .114 & .379 & .433 & .000 \\
\hline TBA $(\mu \mathrm{mol} / \mathrm{L})$ & .172 & .052 & .129 & .146 & .188 & .033 \\
\hline $\mathrm{CHE}(\mathrm{KU} / \mathrm{L})$ & .162 & .087 & -.036 & .707 & .239 & .011 \\
\hline Ada (U/L) & -.010 & .926 & .115 & .293 & -.081 & .458 \\
\hline $\mathrm{LP}(\mathrm{a} ; \mathrm{mg} / \mathrm{L})$ & -.011 & .920 & -.130 & .240 & .060 & .586 \\
\hline APOA (g/L) & .273 & .012 & .086 & .437 & .334 & .002 \\
\hline APOB $(g / L)$ & .233 & .033 & .065 & .559 & .260 & .017 \\
\hline LDL-c (mmol/L) & .136 & .148 & -.027 & .772 & .181 & .054 \\
\hline $\mathrm{HDL}-\mathrm{c}(\mathrm{mmol} / \mathrm{L})$ & .186 & .046 & .017 & .858 & .255 & .006 \\
\hline $\mathrm{TCHO}(\mathrm{mmol} / \mathrm{L})$ & .178 & .057 & -.050 & .598 & .256 & .006 \\
\hline $\mathrm{TG}$ (mmol/L) & .080 & .393 & -.059 & .531 & .136 & .146 \\
\hline $\mathrm{FIB}(\mathrm{g} / \mathrm{L})$ & -.149 & .134 & -.134 & .180 & -.166 & .095 \\
\hline $\lg G(g / L)$ & -.338 & .001 & -.230 & .027 & -.337 & .001 \\
\hline $\lg \mathrm{A}(\mathrm{g} / \mathrm{L})$ & -.023 & .827 & -.002 & .986 & -.072 & .500 \\
\hline $\lg M(g / L)$ & .015 & .892 & .034 & .755 & .022 & .84 \\
\hline C3 (g/L) & .081 & .443 & -.067 & .531 & .141 & .184 \\
\hline $\mathrm{C4}(\mathrm{g} / \mathrm{L})$ & .150 & .158 & .116 & .279 & .117 & .273 \\
\hline RF (KU/L) & -.069 & .462 & -.066 & .487 & -.044 & .645 \\
\hline CRP (mg/L) & -.037 & .689 & .116 & .214 & -.134 & .149 \\
\hline Hypertension & -.107 & .228 & -.003 & .976 & -.112 & .208 \\
\hline Lung disease & -.003 & .975 & -.027 & .762 & .016 & .853 \\
\hline Diabetes & -.103 & .246 & -.020 & .821 & -.120 & .175 \\
\hline Infection & -.071 & .419 & .005 & .951 & -.069 & .440 \\
\hline $\operatorname{NEU}\left(10^{9} / \mathrm{L}\right)$ & .044 & .621 & -.030 & .735 & .062 & .491 \\
\hline $\operatorname{LYM}\left(10^{9} / \mathrm{L}\right)$ & -.012 & .889 & -.146 & .103 & .027 & .763 \\
\hline $\mathrm{ESR}(\mathrm{mm} / \mathrm{H})$ & -.266 & .010 & -.141 & .113 & -.223 & .008 \\
\hline
\end{tabular}


antigenic peptides to the HLA molecular to inhibit the immune response. ${ }^{10}$ Increasing the level of bilirubins in RA patients might be an effective method to control joint inflammation. However, although the therapy effects of bilirubin have been confirmed in a collageninduced arthritis models, the toxicity of high concentrations of bilirubin should be taken into consideration in clinical use.

As we know, indirect bilirubin is not water soluble and bound to albumin in the blood. Consistent with our study, albumin was significantly decreased in RA patients and exhibited a positive association with serum indirect bilirubin. Albumin, another serum antioxidant, has been confirmed inversely associated with activation of proinflammatory cytokines. ${ }^{19,20}$ Hence, we proposed that both lower levels of bilirubin and albumin exerted an additive effect on the disease progression of RA and exacerbated the chronic inflammation. We also detected inverse association between total, indirect bilirubin and ESR, but not any correlation with other inflammatory markers, such as CRP, LYM, and NEU. Interestingly, our present study showed a significantly negative association of serum bilirubin with IgG. This phenomenon might be due to the inhibition of the complement cascade by blocking binding of $\mathrm{C} 1$ complex to antibody and regulating the expression of Fc receptors on macrophages. ${ }^{21}$ But strangely, the correlations between RF, C3, C4, IgA, IgM, and bilirubin have been not detected. The reason remained unknown and might be due to the small samples. Moreover, HDL-C, which binding with the S1P protein to suppress the lymphocyte cells proliferation and inflammatory responses, is significantly correlated with bilirubin.

However, the retrospective cross-sectional design and a singlecenter data in this study should be considered as limitations. Because of lack of follow-up, we could not evaluate the prognostic role of serum bilirubin on RA. To avoid the potential interference of confounding factors, only RA patients without any complications were included. Then, the sample size was relatively small. Therefore, a large sample in the future should be needed to certify the significance in RA patients.

In view of our observation and those from other findings, it is supported that serum bilirubin has a protective effect on RA, since the levels of bilirubin were significantly decreased in RA patients. This suggests that strategies to inducing biosynthesis of bilirubin might be partially preventing the RA progression. However, safe and persistent interventions to induce bilirubin should be taken into consideration.

\section{AUTHOR CONTRIBUTIONS}

All the authors have accepted responsibility for the entire content of this submitted article and approved submission.

\section{REFERENCES}

1. Khojah HM, Ahmed S, Abdel-Rahman MS, Hamza AB. Reactive oxygen and nitrogen species in patients with rheumatoid arthritis as potential biomarkers for disease activity and the role of antioxidants. Free Radic Biol Med. 2016;97:285-291.
2. Yu DH, Yi JK, Yuh HS, et al. Over-expression of extracellular superoxide dismutase in mouse synovial tissue attenuates the inflammatory arthritis. Exp Mol Med. 2012;44:529-535.

3. Pham-Huy LA, He H, Pham-Huy C. Free radicals, antioxidants in disease and health. Int J Biomed Sci. 2008;4:89-96.

4. Remans PH, van Oosterhout M, Smeets TJ, et al. Intracellular free radical production in synovial T lymphocytes from patients with rheumatoid arthritis. Arthritis Rheum. 2005;52:2003-2009.

5. Grimsrud PA, Xie H, Griffin TJ, Bernlohr DA. Oxidative stress and covalent modification of protein with bioactive aldehydes. J Biol Chem. 2008;283:21837-21841.

6. Jikimoto T, Nishikubo $\mathrm{Y}$, Koshiba M, et al. Thioredoxin as a biomarker for oxidative stress in patients with rheumatoid arthritis. Mol Immunol. 2002;38:765-772.

7. Andreev-Andrievskiy AA, Kolosova NG, Stefanova NA, et al. Efficacy of mitochondrial antioxidant plastoquinonyl-decyltriphenylphosphonium bromide (SkQ1) in the rat model of autoimmune arthritis. Oxid Med Cell Longev. 2016;2016:8703645.

8. Neha, Ansari MM, Khan HA. Yohimbine hydrochloride ameliorates collagen type-II-induced arthritis targeting oxidative stress and inflammatory cytokines in Wistar rats. Environ Toxicol. 2016; doi: 10.1002/tox.22264.

9. Jangi S, Otterbein L, Robson S. The molecular basis for the immunomodulatory activities of unconjugated bilirubin. Int J Biochem Cell Biol. 2013;45:2843-2851.

10. Isogai $\mathrm{H}$, Hirayama N. A possible molecular mechanism of immunomodulatory activity of bilirubin. Int J Med Chem. 2013;2013:467383.

11. Yang Z, Liang Y, Li C, Xi W, Zhong R. Bilirubin levels in patients with systemic lupus erythematosus: increased or decreased? Rheumatol Int. 2012;32:2423-2430.

12. dos Santos BH, de R Almeida CM, Skare TL. Systemic lupus erythematosus activity and serum bilirubins. Acta Reumatol Port. 2013;38:242-246.

13. Peng F, Deng $X, Y u Y$, et al. Serum bilirubin concentrations and multiple sclerosis. J Clin Neurosci. 2011;18:1355-1359.

14. Hench PS. Effect of jaundice on rheumatoid arthritis. Br Med J. 1938;2:394-398.

15. Sullivan SN. The effect of jaundice on rheumatoid arthritis. J Rheumatol. 1980;7:417-418.

16. Fischman D, Valluri A, Gorrepati VS, Murphy ME, Peters I, Cheriyath P. Bilirubin as a protective factor for rheumatoid arthritis: an NHANES study of 2003-2006 data. J Clin Med Res. 2010;2:256-260.

17. Liu Y, Li P, Lu J, et al. Bilirubin possesses powerful immunomodulatory activity and suppresses experimental autoimmune encephalomyelitis. J Immunol. 2008;181:1887-1897.

18. Rocuts $F$, Zhang $X$, Yan J, et al. Bilirubin promotes de novo generation of T regulatory cells. Cell Transplant. 2010;19:443-451.

19. Don BR, Kaysen G. Serum albumin: relationship to inflammation and nutrition. Semin Dial. 2004;17:432-437.

20. Garcia-Martinez R, Andreola F, Mehta G, et al. Immunomodulatory and antioxidant function of albumin stabilises the endothelium and improves survival in a rodent model of chronic liver failure. $J$ Hepatol. 2015;62:799-806.

21. Vetvicka V, Fornusek L, Zidkova J. The expression of Fc and complement receptors in young, adult and aged mice. Immunology. 1985;56:73-80.

How to cite this article: Juping D, Yuan Y, Shiyong C, et al. Serum bilirubin and the risk of rheumatoid arthritis. J Clin Lab Anal. 2017;31:e22118. https://doi.org/10.1002/jcla.22118 